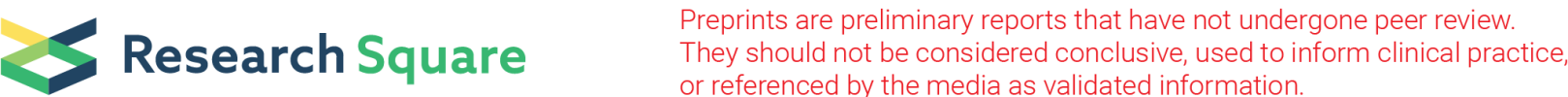

\section{Preoperative pain aggravates postoperative cognitive deficits and hippocampal neuroinflammation in rats}

\section{Xiang Gao}

Fujian Provincia Maternity and Children's Hospital

\section{Xian Ding}

Fujian Provincial Maternity and Children's Hospital

\section{Huan Yi}

Fujian Provincial Maternity and Children's Hospital

\section{Chuan-tao Lin}

Fujian Provincial Maternity and Children's Hospital

\section{Yu-ping Wang}

Fujian Provincial Maternity and Children's Hospital

\section{De-biao Yu}

Fujian Provincial Hospital

Jian-ying Yan ( $2474157071 @ q q . c o m$ )

Fujian Provincia Maternity and Children's Hospital https://orcid.org/0000-0003-1520-7349

\section{Research article}

Keywords: Preoperative pain, Cognitive function, PND, Acetylcholine

Posted Date: December 3rd, 2019

DOI: https://doi.org/10.21203/rs.2.17941/v1

License: (c) (1) This work is licensed under a Creative Commons Attribution 4.0 International License. Read Full License 


\section{Abstract}

Background Perioperative neurocognitive disorder (PND) is the progressive deterioration of cognitive function after surgery. The purpose of this study was to observe the effect of preoperative pain on inflammatory factors and neuronal apoptosis in the hippocampus of rats.

Methods 36 adult male Sprague-Dawley rats were randomly divided into 4 groups: the control group, the pain group, the pain+operation group, and the operation group. 6 days before the surgery, the rats received cognitive training, and the cognitive evaluation was carried out on the 1,3 and 7 th days after the surgery. The rats were killed on the first, third and seventh days after the surgery ( $n=3$ rats/day). The cognitive function of rats was evaluated by the Morris Water Maze (MWM), and the expression levels of the pro-inflammatory cytokines interleukin $6(\mathrm{IL}-6)$, Interleukin $1 \beta(\mathrm{IL}-1 \beta)$ and Tumor Necrosis Factor-a(TNFa), Acetylcholine(Ach)and Cyclic Adenosine monophosphate(cAMP), protein kinase A(PKA)and gammaaminobutyric acid type $A$ receptors(GABAA) in the hippocampus were measured on the $1 \mathrm{st}, 3 \mathrm{rd}$ and 7 th days after the operation.

Results Our results showed that the pain model rats exhibited impaired behavior on the first day $(\mathrm{P}<$ 0.001 ), and this lasted until the 7th day after the operation ( $P \leq 0.002$ and $P \leq 0.001$, respectively). Preoperative pain model rats showed a higher level of apoptosis than that shown by the simple operation rats. On the 1st, 3rd and 7th days after the operation, the protein content of IL-1 $1 \beta$, IL- 6 and TNF- $\alpha$ in the pain operation group was increased compared to that in the simple operation group $(P<0.001)$. ACh, cAMP, PKA and GABAA expression in the hippocampus was decreased after operation in the preoperative pain model rats.

Conclusion Preoperative pain is a key risk factor for the development of PND. The ACh-PKA-GABAA signaling pathway plays a key role in the acetylcholine pathway.

\section{Background}

Perioperative neurocognitive disorder (PND) includes acute delirium and persistent postoperative cognitive impairment [1]. Because PND leads to an increase in postoperative mortality, an increase in complications and the prolongation of hospitalization, it is more likely to evolve into Alzheimer's disease $[2,3]$, which has attracted increasing attention from diverse disciplines. However, the mechanism of PND has not been clearly elucidated yet. Therefore, preventing and treating controllable risk factors for PND have become important methods of managing PND. Clinical observation has shown that the incidence of POCD in patients with preoperative pain is higher than that in patients with a pain duration $\geq 3$ months $[4,5]$. Animal experiments have also shown that preoperative chronic pain facilitates a decrease in cognitive function in rats [6]. These results suggest that preoperative chronic pain may be an important controllable risk factor for PND. Therefore, it may be critical for managing PND and studying the mechanism underlying preoperative slow pain and PND. 
Rodent models and clinical data have shown that neuroinflammation plays a key role in the pathogenesis of PND [7]. Increasing evidence has suggested that chronic pain plays an important role in inducing and maintaining neuroinflammation induced and maintained by immunocyte infiltration [8], glial cell activation [9] and central nervous system inflammatory mediators [9-12]. Peripheral pro-inflammatory cytokines, which are mainly involved in the inflammatory regulation of acetylcholine through the vagus nerve reflex arc, can be invoked as primary initiators of neuroinflammation [13]. This process involves the pro-inflammatory cytokines IL-1 $1 \beta, I L-6$, and TNF- $\alpha$ [14-15]. Acetylcholine receptors can activate cyclic adenosine monophosphate (CAMP)-protein kinase $A(P K A)$ through $G$ protein coupling. The second messenger pathway [16] leads to the phosphorylation of the $G A B A_{A}$ receptor. The $G A B A_{A}$ receptor is an inhibitory neurotransmitter mainly found in the central nervous system [17]. $G A B A_{A}$ is involved in the cognitive development of rats and strictly controls spatial learning [18].

Although it has been confirmed that preoperative chronic pain maintains the balance of inflammatory mediators in the central nervous system through the acetylcholine pathway, it is unclear whether this balance is disrupted after surgical and anesthetic stimulation, resulting in PND. The purpose of this study was to evaluate inflammatory factor expression and neuronal apoptosis in the hippocampus of rats with preoperative chronic pain to determine the correlation between chronic pain and PND, detect the contents of ACh, CAMP, PKA and $\mathrm{GABA}_{A}$, and observe the effect of preoperative chronic pain on the acetylcholine pathway.

\section{Methods}

\section{Animals}

All the animals used in this study are from the experimental animal center of Fujian maternal and child health care institute, and are raised according to its guidelines. All experiments were performed in the experimental animal center of Fujian maternal and child health care institute. All animals were housed in an environment with a temperature of $24^{\circ} \mathrm{C}$ to $25^{\circ} \mathrm{C}, 12$ hours light and 12 hours dark cycle, free access to food and water. All rats were acclimated to their environment for at least 7-10 days prior to the experiment. In this study, all animal-related experiments were carried out under the revised guidelines for Nursing and Use of Experimental Animals [19] (American Society of Physiology) and were approved by the Ethics Committee of our hospital (NO. 2018-093).

\section{Groups and Experimental steps}

36 adult male Sprague-Dawley rats (3-6 months), weighing 350-400 g, were randomly by random number table according to body weight divided into 4 groups: the control group, the pain group, the pain+operation group and the operation group. Control animals received aseptic saline to control for the effect of injection stress. The right posterior foot pad of each mouse in the pain group, was subcutaneously injected with complete Freund's adjuvant $20 \mu \mathrm{l}$. The mice in the pain+operation group received the same treatment as those in the pain group and underwent partial hepatectomy with general 
anesthesia. In short, the liver was exposed through a 1-2 cm midline abdominal incision, and the left lobe of the liver (equivalent to approximately $30 \%$ of the organ) was resected. The wound was then permeated with $0.25 \%$ lidocaine and closed with an aseptic suture. The mice in the simple operation group underwent hepatectomy only (the operation procedure was the same as that in the pain operation group).

Prior to surgical modeling, the rats underwent 6 days of the morris water maze (MWM) training. After MWM were conducted on days 1,3 , and 7 after surgery.

\section{Tissue preparation}

The rats were killed on days 1, 3 and 7 ( $n=3$ per day) after surgery. The rats were decapitated under anesthesia with $10 \%$ pentobarbital sodium, the brains were removed and the hippocampi were extracted. The separated hippocampi were separated into two parts; one part was stored at $80^{\circ} \mathrm{C}$ for preservation and later use, and the other was fixed with polyformaldehyde (PFA).

\section{Cognitive testing}

The Morris water maze is a hippocampal-dependent spatial learning test for rodents [20]. The rats were trained with the platform in a fixed position and tested three times a day for 6 days. Preoperative pain was first induced in the animals, and then partial hepatectomy was performed on day 7. On the 1st, 3rd and 7th days after the operation, the rats were subjected to reverse testing, in which the platform was moved to the outer edge of the pool. The swimming distance, speed and latency for the rats to find the platform were recorded by a video system attached to the ceiling, and the digital images were analyzed by water maze software (HVS image, UK).

\section{ELISA}

The levels of ACh, CAMP, IL-1, IL-6 and TNF-1 in the samples were determined according to the instructions of the kit. The results were analyzed using TMB, which is converted to blue by the catalysis of peroxide and then to yellow by the action of an acid. Changes in color were positively correlated with ACh, CAMP, IL $-1 \beta$, IL -6 and TNF- $\alpha$ levels in the samples. The absorbance (OD value) was measured at a wavelength of $450 \mathrm{~nm}$ by an enzyme labeling instrument, and the concentrations in the sample were estimated. The supernatant was used for the determination of the total protein level with ELISA kits (Dakewe, Shenzhen, China).

\section{Western blot analysis}

Hippocampal tissue samples were homogenized in cold lysis buffer containing protease inhibitors and then centrifuged. Standardized protein samples were boiled in buffer for 5 minutes, separated on a polyacrylamide microgel and transferred to a nitrocellulose membrane by electrophoresis. The membrane was blocked in TBS for 1 hour with $5 \%$ defatted milk and incubated in rabbit anti-GABA $A$ (1:1000)( merck $^{2}$ Millipore, USA) and anti-PKA antibodies (1:1000) (merck Millipore, USA)overnight at $4^{\circ} \mathrm{C}$. the membrane 
was placed in TBS with secondary antibody diluted 1:400 for 1 hour at room temperature. Protein visualization was performed by using an enhanced chemiluminescence detection kit (ECL; Amersham Biosciences). The relative expression levels of the target proteins were standardized to the expression level of $\beta$-actin.

\section{Immunohistochemistry}

Seven days after the operation, the PFA-fixed hippocampi were embedded in paraffin wax after dehydration in graded ethanol solutions. A slicing machine was used to slice the paraffin-embedded tissues into 4- $\mu \mathrm{m}$ thick continuous coronal sections. Then, the hydrated sections were stained in hematoxylin and eosin (HE) and dehydrated in ethanol. The stained sections were removed and used to observe the morphology and arrangement of neurons in the rat hippocampus by microscopy.

\section{TUNEL}

Seven days after surgery, the sections were fixed in 4\% PFA, incubated, and then washed and developed using diaminobenzoic acid reagent. The sections were counterstained with Harris hematoxylin, and five high power fields from each section were analyzed by microscopy. In each field of view, 100 cells were selected to calculate the average ratio of apoptotic cells to determine the apoptotic index (Al).

\section{Statistical analysis}

Two-way ANOVA was used to analyze the immunohistochemical, ELISA and Western blot data. Repetitive two-way ANOVA was used to analyze training behavior. Separate two-way ANOVA was used to examine the effects of pain and surgery on working memory performance in the reversal test. When ANOVA was significant, the correlation between MWM parameters and pro-inflammatory cytokine expression was analyzed by an independent sample $t$ test. $\mathrm{P}<0.05$ was considered to be statistically significant.

\section{Results}

Preoperative pain aggravates spatial learning and memory impairment after surgical trauma in rats

The results of the MWM test showed that the distance traveled and latency to find the platform were decreased in the simple operation group and the pain operation group on the first day after operation compared with those in the blank group and the simple pain group (Fig. $1 \mathrm{~A}$ and B). Compared with the other three groups, the pain operation group showed the same cognitive impairment on the 3rd and 7th days after operation (Fig. 1A and B). Compared with those of the pain operation group on the 3rd day after operation, the distance and latency parameters of the simple operation group were significantly improved, but they were still lower than those of the blank group and the simple pain group; the above data were improved until 7 days after operation (Fig. 1A and B). These results showed that preoperative pain and hepatectomy damage spatial learning and memory and induce a prolonged disease response in rats. 
To study the apoptosis of neurons in the hippocampal area of the rats with preoperative pain, we used $\mathrm{HE}$ staining to observe the morphology of the neurons in the hippocampal CA1 region of the rats. Blank group cells were closely arranged, and the membranes were transparent. However, the simple operation group and the pain operation group showed pyramidal cells with agglomerated nuclei, wider cell gaps, and cell disorder, necrotic neurons and the loss of a large number of neuronal cells. Compared with those in the pain operation group, the cells in the operation group and the pain group exhibited significantly improved neuronal morphology and arrangement (Fig. 2).

In addition, the results of TUNEL staining are shown in Figure 3. Higher Al and significant neuronal apoptosis were found in the hippocampal CA1 region of the rats in the pain+operation group.

Expression of inflammatory cytokines after surgical trauma in the hippocampus of rats with preoperative pain

To study whether pain and surgical trauma change the expression of pro-inflammatory factors in the hippocampus, the protein contents of IL-1 $\beta$, IL- 6 and TNF- $a$ were measured by ELISA at different time points. On the 1st, 3rd and 7th days after the operation, the protein contents of IL-1 $\beta$, IL- 6 and TNF- $\alpha$ in the pain operation group were increased compared with those in the other three groups (Fig. 4). The protein contents of IL $-1 \beta, I L-6$ and TNF- $\alpha$ in the operation group were increased on the 1 st, 3rd and 7th days after the operation compared with those in the blank group and pain group. There was no significant change in the protein contents in the blank group and simple pain group on the $1 \mathrm{st}$, 3rd or 7 th days after the operation. These results suggest that preoperative pain is more likely to induce the longlasting expression of pro-inflammatory factors in the hippocampus after operation and that surgery is also induces the expression of pro-inflammatory factors in the hippocampus.

The acetylcholine pathway was activated in rats with preoperative pain exposed to surgical trauma.

To understand the effect of preoperative pain on acetylcholine pathway activation, a further study was conducted. The expression of ACh, CAMP, PKA, GABA $A$ and other proteins in the brain was determined by

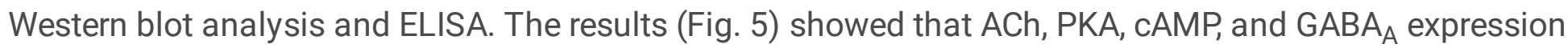
decreased significantly on the 7th day after partial hepatectomy in rats with preoperative pain compared with that in the rats from the other three groups. In addition, the protein contents of ACh, PKA, CAMP, and $\mathrm{GABA}_{A}$ in the simple operation group were significantly lower than those in the simple pain group and the blank group on the 1st and 3rd day but higher on the 7th day. The levels were clearly restored. This suggests that cognitive impairment after operation may be due to the inhibition of the ACh signaling pathway, which correlated with PKA and $\mathrm{GABA}_{A}$ expression, and that the presence of pain before operation increases the likelihood and prolongs this effect.

\section{Discussion}


The results of our study showed that the distance and latency parameters of the water maze decreased more significantly in the group with preoperative chronic pain than in the simple operation group. The

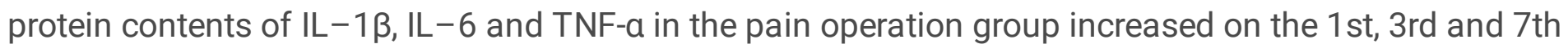
days after the operation, indicating that preoperative chronic pain can significantly aggravate the neuroinflammatory reaction after operation. Preoperative chronic pain leads to neuronal apoptosis and increases the expression of pro-inflammatory cytokines in the hippocampus, which leads to a decrease in cognitive function after operation. Our study revealed that rats with preoperative pain are susceptible to cognitive impairment after surgical trauma. Postoperative cognitive impairment paralleled the increased expression of pro-inflammatory cytokines in the central nervous system.

In this study, we aimed to observe the effect of preoperative chronic pain on postoperative cognitive impairment, so we chose complete Freund's adjuvant (CFA) to establish a chronic inflammatory pain model. CFA can precipitate antigens locally that releases slowly and plays an important role in stimulating antigens. It is an ideal model of chronic pain that is widely used. For our surgical intervention, we chose partial liver surgery. Distribution of painless nerves in the liver can minimize the impact of visceral pain on the central system during and after the operation and the interference between different pains. The liver acts as a machine. As the main metabolic organ of the body, the liver plays a key role in drug metabolism. However, there was no drug intervention in this study, so the metabolic activity of the liver had little effect on this experiment. In addition, we chose to remove $30 \%$ of the liver because such a procedure has no impact on the normal physiological function of the liver. Therefore, partial liver surgery was used as an intervention to study the effect of preoperative chronic pain on postoperative cognitive function.

Our initial results showed that, compared with those of the blank group and the simple pain group, the distance and latency parameters in the water maze test of the operation group were decreased; however, the rats with preoperative pain showed poorer cognitive ability than that of the rats that underwent simple operation, showing that the distance and latency parameters of the MWM experiment were prolonged. It has been suggested that preoperative chronic pain is an important risk factor for decreases in cognitive and learning abilities in rats after operation and that it prolongs the disease response. It is well known that preoperative pain activates the peripheral innate immune system. Peripheral immune attacks are transmitted to the brain through a variety of humoral and neurological pathways [22]. This immune system-brain signal leads to the production of pro-inflammatory cytokines in the brain, which mainly involves the activation of glial cells [23]. The activation of the peripheral innate immune system promotes the release of inflammatory cytokines, such as IL $-1 \beta, I L-6$ and TNF- $a$, by directly or indirectly activating microglia [24]. These neurotoxic inflammatory mediators can further change the function of neurons and lead to a decrease in cognitive function [25]. Therefore, we evaluated the expression of pro-inflammatory cytokines in the hippocampus of the rats. The results showed that the expression of IL-1 $\beta$, I L6 and TNFa in the hippocampus of the operation group was increased significantly $(P<0.05)$ compared with that in the control group and pain group. In rats with preoperative pain, IL-1 3 , IL6 and TNFa were more highly expressed in the hippocampus of rats $(P<0.05)$. This suggests that preoperative pain can cause a 
significant inflammatory response in the hippocampus after operation, so we further determined that preoperative pain is an important risk factor for postoperative cognitive impairment[21].

In our study, it was found that rats with preoperative chronic pain had longer reaction times than those that underwent simple surgery, which is an important phenomenon that was not mentioned in previous studies. We speculate that this may be related to the effect of acetylcholine. In some in vitro experiments, it has been shown that the inflammatory reaction of microglia can be inhibited by nicotine, while nicotine can reduce the local inflammatory reaction through the acetylcholine pathway and produce neuroprotective effects [26]. Therefore, we further observed the content of ACh in the hippocampus. The results showed that, compared with the blank group and the simple pain group, the pain+operation group. exhibited significantly decreased ACh levels in the hippocampus on the 3rd and 7th days. ACh levels in the hippocampal region decreased only on the 1st and 3rd days after the operation in the operation group $(P<0.05)$, while the ACh levels in the hippocampus decreased significantly on the 3rd and 7 th days after the operation $(P<0.05)$, and the levels in the operation group decreased only on the 1 st and 3rd days after the operation $(P<0.05)$. According to the results, we speculate that, although some microglia may be activated in rats with chronic pain, there is no neuroinflammatory reaction, and cognitive function is not affected due to the anti-inflammatory pathway of acetylcholine. In rats that underwent simple operation, we observed that IL-1 1 , IL-6 and TNF- $a$ expression increased to varying degrees, indicating that surgery is a risk factor for PND, which is consistent with the findings of previous studies. Like [25]. However, as time passed after the operation, the expression of inflammatory factors in the rats in the simple operation group gradually decreased, indicating that the operation may be a transient stimulus that induces an increase of the expression of pro-inflammatory cytokines over a short period of time and that as time passes, acetylcholine increases, the anti-inflammatory effect increases, and cognitive function gradually recovers. However, there was no significant decrease in preoperative pain in rats under the same conditions, which indicates that the combined effects of preoperative chronic pain and surgery can induce a high level of neuronal apoptosis and prevent the body from repairing itself. Therefore, we think that preoperative pain is a major factor that leads to the prolongation of PND.

We further observed the activation of the acetylcholine pathway and its $\mathrm{G}$ protein coupling in the hippocampus. The results showed that the expression of cAMP, PKA, and $G_{A B A}$ in the hippocampus of rats that underwent partial hepatectomy was significantly lower than that in rats that did not undergo operation, and the content of AMP, PKA, and $\mathrm{GABA}_{A}$ in the hippocampus of rats with preoperative pain was significantly lower than that of rats that underwent simple operation $(P<0.05)$. Additionally, the content of AMP, PKA, and $G_{A B A}$ in the hippocampus of rats with preoperative pain was significantly higher than that in rats that did not undergo operation $(P<0.05)$, and the content of PKA in the hippocampus of rats with preoperative pain was significantly lower than that of rats that did not undergo operation $(P<0.05)$; this was more obvious on the 7th day after the operation. The acetylcholine receptor can activate the CAMP-PKA pathway through $G$ protein coupling to intracellular CAMP-activated PKAphosphorylated CREB ( $p$-CREB), and activated p-CREB can also interact with the targeted CRE region to regulate the transcription of many downstream genes, promote the development of synaptic connections, 
and form a long-term memory. This process is related to synaptic plasticity. Synaptic plasticity refers to the adjustment of the connection strength between nerve cells [27] An activity-dependent increase in synaptic transmission intensity may result in long-term potentiation (LTP)[28], and memories are considered to be generated through the process of LTP, which is generally regarded as one of the main molecular mechanisms that form the basis of learning and memory. Interestingly, studies have shown that pro-inflammatory cytokines involved in immuno system-brain signal transduction are thought to destroy synaptic plasticity [14]. Downstream of the cAMP-PKA pathway, $G_{A B A}$ can gate the $G_{A B A}$ receptor signal in synaptic transmission during LTP induced by high-frequency activity, modulate the frequency dependence of synaptic plasticity [30] and play a key role in learning and memory. Based on the above information, we speculate that $G_{A B A}$ is affected by the ACh-CAMP-PKA signaling pathway and that the role of the ACh-CAMP-PKA pathway is very important for the development of PND. Therefore, it can be inferred that preoperative chronic pain leads to inflammation in the central nervous system, activates the acetylcholine anti-inflammatory pathway, maintains the balance between the internal environment and synaptic plasticity, and does not induce obvious cognitive impairment. After surgical stimulation, neuroinflammation further increases, destroys the original balance, and leads to the release of a large number of central inflammatory, the induction of high levels of apoptosis in neuronal cells, and acetylcholine production disorder. The anti-inflammatory effect is weakened, and the ACh-cAMP-PKA signal is passed through the shadow pathway at the same time. Reducing $G_{A B A}$ results in the disruption of synaptic plasticity, which leads to an increase in PND incidence and prolongation of PND.

There are some shortcomings in this study. In our study, the behavior, apoptosis level of hippocampal cells, and expression of pro-inflammatory cytokines and acetylcholine pathway proteins were compared between rats with preoperative chronic in the presence and absence of surgery but not between rats with preoperative chronic pain, so it is impossible to determine whether the rats had preoperative chronic pain. The effective management of preoperative chronic pain has an effect on postoperative cognitive function, and whether the peripheral innate immune system is activated by preoperative chronic pain, especially whether "immune memory ability" occurs in this process, is important. At the same time, the effects of preoperative chronic pain and surgical trauma pain on the consequences were not eliminated. In the study of the cellular pathway, we observed changes in the expression of different proteins in the pathway, but unfortunately, we did not determine the specific proteins. At the same time, we did not elucidate the changes in endogenous and exogenous acetylcholine, and we did not inhibit the pathway to determine the effect of the pathway. Therefore, this study is only a preliminary observational study, and our follow-up study will compensate for the shortcomings.

\section{Conclusions}

In conclusion, the results of this study suggest that pro-inflammatory cytokines may play an important role in disrupting the normal cognitive function of the hippocampus. Preoperative pain leads to an excessive and prolonged neuroinflammatory response, which involves the acetylcholine pathway, in which the ACh-PKA-GABA $A_{A}$ cell signaling pathway plays an essential role. Additionally, preoperative pain 
is a key risk factor for the development of PND. Preoperative pain management and inflammatory inhibition may be promising strategies for preventing PND.

\section{List Of Abbreviations}

PND: Perioperative neurocognitive disorder; MWM: Morris Water Maze; IL-6: Interleukin-6; IL-1ß: Interleukin-1 $\beta$; TNF-a: Tumor Necrosis Factor-a; Ach: Acetylcholine; cAMP: Cyclic Adenosine monophosphate; PKA: protein kinase $A ; G_{B B A}$ : gamma-aminobutyric acid type $A$ receptors; PFA: polyformaldehyde; CFA: Freund's adjuvant; LTP: long-term potentiation.

\section{Declarations}

\section{Ethics approval and consent to participate}

All animals used in this study were reared in accordance with the guidelines of the Laboratory Animal Center of Fujian Maternal and Child Health Hospital and approved by the Ethics Committee of Fujian Maternal and Child Health Hospital (NO. 2018-093).

\section{Consent for publication}

Not applicable.

\section{Availability of data and materials}

The data that support the findings of this study in form of Excel files are available from the corresponding author.

\section{Competing interests}

The authors declare that they have no competing interests.

\section{Funding}

This study is currently receiving grants from Fujian Provincial Maternity and Children's Hospital Science and Technology Innovation Initiation Fund 2018(No. FuyouYCXM 18-23). The funding bodies did not have any influence on the design of the study, collection, analysis and interpretation of data and writing the manuscript.

\section{Authors' contributions}


$X G$ and $X \mathrm{D}$ contributed to the design of the research and interpretation of the data equally and should be regarded as co-first authors. HY, CTL and YPWang performed the animal experiments and collected data. DBY performed Molecular biological parts of the study and analyzed the data and cytokines. JYY supervised the project. All authors read and approved the final manuscript.

\section{Acknowledgements}

We thank members of the laboratory for technical help.

\section{References}

1. Evered L. ,Silbert B., Knopman DS, Scott DA, DeKosky ST, Rasmussen LS, Oh ES Crosby G., Berger M., Eckenhoff RG, Evered Lisbeth, Eckenhoff Roderic G., Ames David, Bekker Alex, Berger Miles, Blacker Deborah, Browndyke Jeffrey, Crosby Greg, Deiner Stacie G., van Dijk Diederik, DeKosky Steven T., Eckenhoff Maryellen F., Eriksson Lars, Galasko Dougas, Hogan Kirk, Inouye Sharon, Knopman David, Lyketsos Constantine, Marcantonio Edward, Maruff Paul, Maze Mervyn, Oh Esther Orser Beverley A., Ottens Thomas, Price Catherine, Rasmussen Lars S., Sachdev Perminder, Schenning Katie, Scott David A., Seiber Frederick E., Silbert Brendan, Silverstein Jeff, Steinmetz Jacob, Terrando Niccolo, Trzapacz Paula, Whittington Rob and Xie Zhongcong. Recommendations for the Nomenclature of Cognitive Change Associated with Anaesthesia and Surgery-2018. British Journal of Anaesthesia. 2018;121(5): 1005-1012. https://doi.org/10.1016/j.bja.2017.11.087

2. Avelino-Silva TJ, Campora F, Curiati JA and Jacob-Filho W. Association between delirium superimposed on dementia and mortality in hospitalized older adults: a prospective cohort study. PLoS Med. 2017;14:e1002264. https://doi.org/10.1371/journal.pmed.1002264

3. Rudolph JL and Marcantonio ER. Review articles: postoperative delirium: acute change with longterm implications.Anesth Analg. 2011;112(5):1202-

1211. https://doi.org/10.1213/ANE.0b013e3182147f6d

4. Bian Huixian Du Hailiang, Wang Jiao and Wang Jun. Effects of preoperative pain duration on early postoperative cognitive dysfunction after joint replacement operations. Practical Pharmacy and Clinical Remedies. 2015;18(4):421-424. https://doi.org/10.14053/j.cnki.ppcr.201504013

5. Zhang Jufeng and Yu Hongbo. Risk factors of early cognitive dysfunction in hip-replacement surgery: a nested case-control study. Laboratory Medicine and Clinical. 2016;13(15): 2146-2148. https://doi.org/3969/j.issn.1672-9455.2016.15.028

6. Chen Sihan, Yee Wei LIM, Wen Daxiang and Yu Weifeng. Preoperative chronic pain facilitates postoperative cognitive declination and its influence on tryptophan hydroxylase in dorsal raphe of tar. Shanghai Medical Journal. 2016; 39(6): 368-373.

7. Subramaniyan S and Terrando N. Neuroinflammation and Perioperative Neurocognitive Disorders. Anesth Analg. 2019;128(4):781-788. https://doi.org/10.1213/ANE.0000000000004053 
8. Costigan M, Moss A, Latremoliere A, Johnston C, Verma-Gandhu M, Herbert TA, Barrett L, Brenner GJ, Vardeh D, Woolf $\mathrm{CJ}$ and Fitzgerald M. T-cell infiltration and signaling in the adult dorsal spinal cord is a major contributor to neuropathic pain-like hypersensitivity. J Neurosci. 2009;29(46):14415-14422. https://doi.org/10.1523/JNEUROSCl.4569-09.2009

9. Ji RR, Berta T and Nedergaard M. Glia and pain: Is chronic pain a gliopathy? Pain. 2013;154(Suppl 1): S10-28. https://doi.org/10.1016/j.pain.2013.06.022

10. Grace PM, Hutchinson MR, Maier SF and Watkins LR. Pathological pain and the neuroimmune interface. Nat Rev Immunol.2014;14(4):217-31. https://doi.org/10.1038/nri3621

11. Milligan ED, Soderquist RG, Malone SM, Mahoney JH, Hughes TS, Langer SJ, Sloane EM, Maier SF, Leinwand LA, Watkins LR and Mahoney MJ. Intrathecal polymer-based interleukin-10 gene delivery for neuropathic pain. Neuron Glia Bi 2006;2(4):293-

308. https://doi.org/10.1017/S1740925X07000488

12. Sloane EM, Soderquist RG, Maier SF, Mahoney MJ, Watkins LR and Milligan ED. Long-term control of neuropathic pain in a non-viral gene therapy paradigm. Gene Ther. 2009;16(4):470-5. https://doi.org/10.1038/gt.2009.21

13. Ali Shah S, Ullah I, Lee HY and Kim MO. Anthocyanins protect against ethanol-induced neuronal apoptosis via gabab1 receptors intracellular signaling in prenatal rat hippocampal neurons. $\mathrm{Mol}$ Neurobiol. 2013;48(1):257-69. https://doi.org/10.1007/s12035-013-8458-y.

14. Di Filippo M, Sarchielli P, Picconi B and Calabresi P. Neuroinflammation and synaptic plasticity: theoretical basis for a novel, immune-centred, therapeutic approach to neurological disorders. Trends Pharmacol Sci. 2008; 29:402-12. https://doi.org/1016/j.tips.2008.06.005

15. Lynch MA. Long-term potentiation and memory. Physiol Rev. 2004;84(1):87-136. https://doi.org/1152/physrev.00014.2003

16. Qiu Y, Wang Y, Wang $X$, Wang $C$ and Xia ZY. Role of the hippocampal 5-HT1A receptor-mediated CAMP/PKA signalling pathway in sevoflurane-induced cognitivedysfunction in aged rats. Journal of International Medical Research, 2018;46(3):1073-1085. https://doi.org/1177/0300060517744037.

17. Fritschy, Jean-Marc and Panzanelli P. GABAA receptors and plasticity of inhibitory neurotransmission in the central nervous system. European Journal of Neuroscience. 2014;39(11):1845-1865. https://doi.org/1111/ejn.12534

18. Deidda G , Parrini M, Naskar S, Bozarth IF, Contestabile A and Cancedda L. Reversing excitatory GABAAR signaling restores synaptic plasticity and memory in a mouse model of Down syndrome[J]. Nature Medicine. 2015;21(4):318-326. https://doi.org/1038/nm.3827.

19. Bayne K. Revised Guide for the Care and Use of Laboratory Animals available. American Physiological Society. 1996;39(4):199, 208-211.

20. Higaki, A., Mogi M, Iwanami J, Min LJ, Bai HY, Shan BS, Kukida M, Kan-No H, Ikeda S, Higaki J and Horiuchi M. Predicting outcome of Morris water maze test in vascular dementia mouse model with deep learning. Plos One. 2018;13(2):e0191708. https://doi.org/1371/journal.pone.0191708. 
21. Rice, D. A., Kluger MT, McNair PJ, Lewis GN, Somogyi AA, Borotkanics R, Barratt DT and Walker M. Persistent postoperative pain after total knee arthroplasty: a prospective cohort study of potential risk factors. British journal of anaesthesia. 2018;121(4): 804-812. https://doi.org/1016/j.bja.2018.05.070.

22. Totsch, Stacie K. and Robert E. Sorge. Immune system involvement in specific pain conditions. Molecular pain. 2017;13: 1744806917724559. https://doi.org/1177/1744806917724559

23. Keunen, Kristin, Ruurd M. van Elburg and Frank van Bel, Manon J. N. L. Benders. Impact of nutrition on brain development and its neuroprotective implications following preterm birth. Pediatric research.2015;77(1-2): 148-155. https://doi.org/1038/pr.2014.171

24. Matt, Stephanie M., and Rodney W. Johnson. Neuro-immune dysfunction during brain aging: new insights in microglial cell regulation. Current opinion in pharmacology.2016;26 (18): 96-101. https://doi.org/1016/j.coph.2015.10.009

25. Calsolaro, Valeria and Paul Edison. Neuroinflammation in Alzheimer's disease: current evidence and future directions. Alzheimer's \& Dementia.2016;12(6): 719-732. https://doi.org/1016/j.jalz

26. Hoover DB. Cholinergic modulation of the immune system presents new approaches for treating inflammation. Pharmacol Ther. 2017;179:1-16. https://doi.org/1016/j.pharmthera.

27. Hegde AN. Proteolysis, synaptic plasticity and memory. Neurobiol Learn Mem. 2017;138:98-110. https://doi.org/10.1016/j.nlm.2016.09.003

28. Palacios-Filardo $\mathrm{J}$ and Mellor JR. Neuromodulation of hippocampal long-term synaptic plasticity. Curr Opin Neurobiol. 2019;54:37-43. https://doi.org/10.1016/j.conb.2018.08.009

29. Hayama T and Kasai H. A new role of GABA on synapses. Brain Nerve. 2014;66(8):987-93.

30. Lyons H R, Land MB, Gibbs TT, Farb DH. Distinct signal transduction pathways for GABA-induced GABAA receptor down-regulation and uncoupling in neuronal culture: a role for voltage-gated calcium channels. Journal of Neurochemistry. 2010;78(5):1114-1126. https://doi.org/10.1046/j.1471-4159.2001.00501.x

31. Pan B, Lian J, Huang XF and Deng C. Aripiprazole Increases the PKA Signalling and Expression of the GABAA Receptor and CREB1 in the Nucleus Accumbens of Rats. Journal of Molecular Neuroscience Mn. 2016;59(1):36- 47. https://doi.org/10.1007/s12031-016-0730-y

\section{Figures}



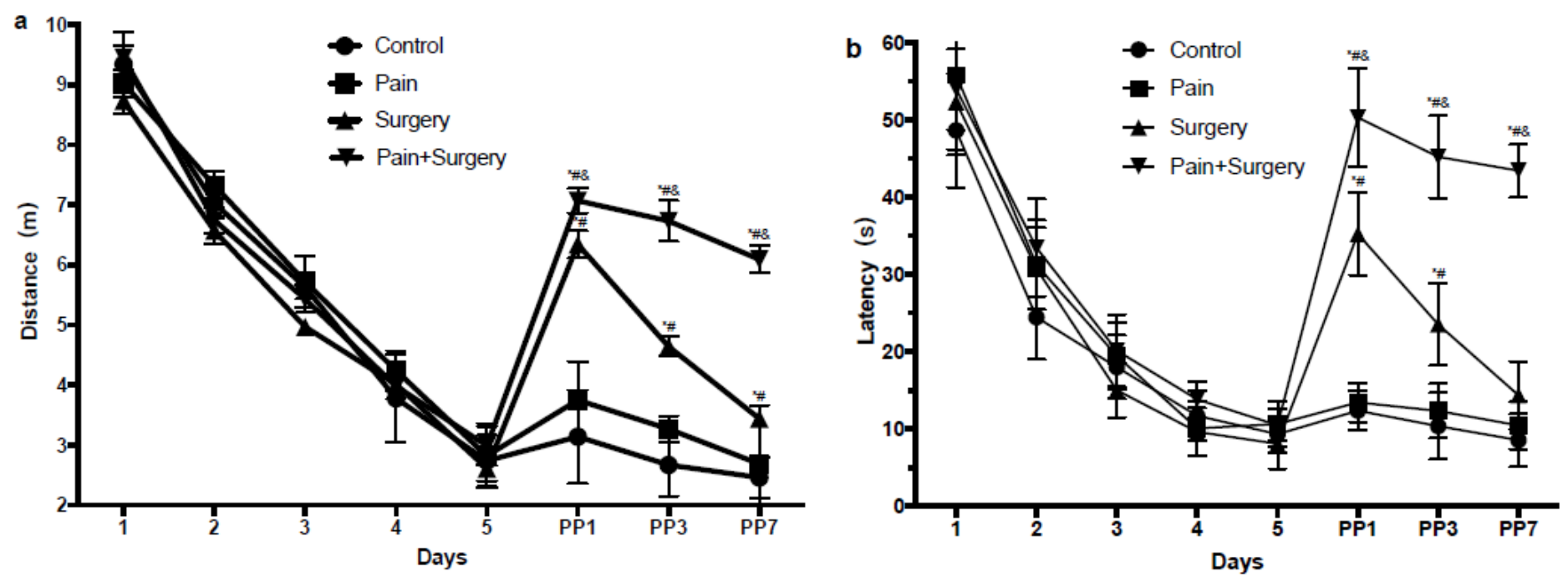

Figure 1

Water maze data of the rats in each group that were trained for 6 days and 7 days after operation. (a) The distance swam to reach the platform on the training day, (b) the latency to reach the platform on the training day and the time swum to reach the platform on the training day. The results are presented as the mean \pm standard deviation. ${ }^{*} \mathrm{P}<0.05$, compared with the blank control group; $\# \mathrm{P}<0.05$ and the simple pain group; \& $\mathrm{P}<0.05$ compared with the simple operation group. Control: control group; pain: pain group; surgery: operation group; pain surgery: pain+operation group; $1,2,3,4,5$, and 6 : training days $1,2,3,4,5$, and 6; pp1, pp3, and pp7: 1, 3, and 7 days after the operation. 


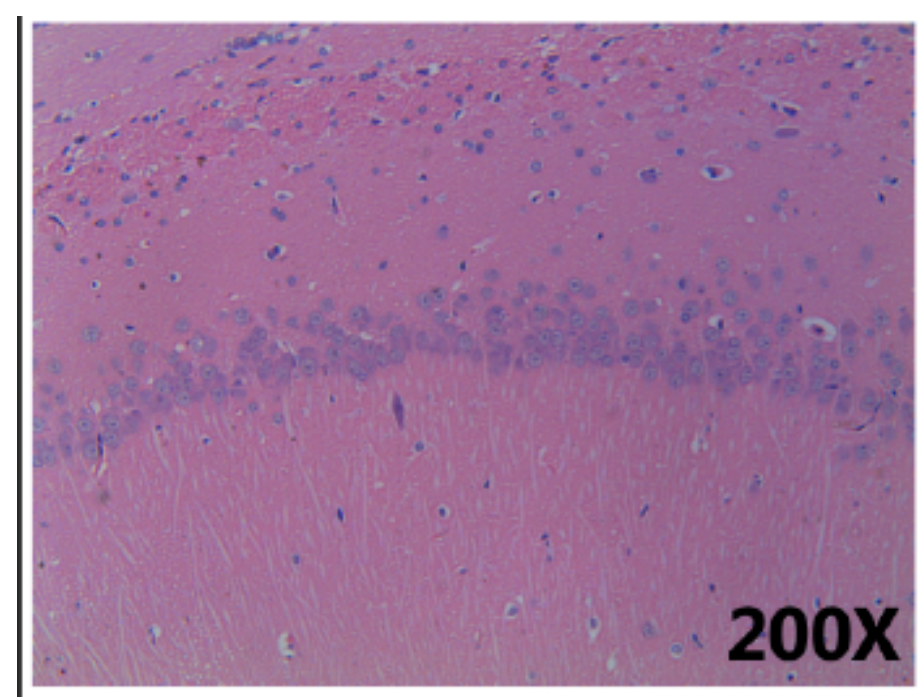

Control

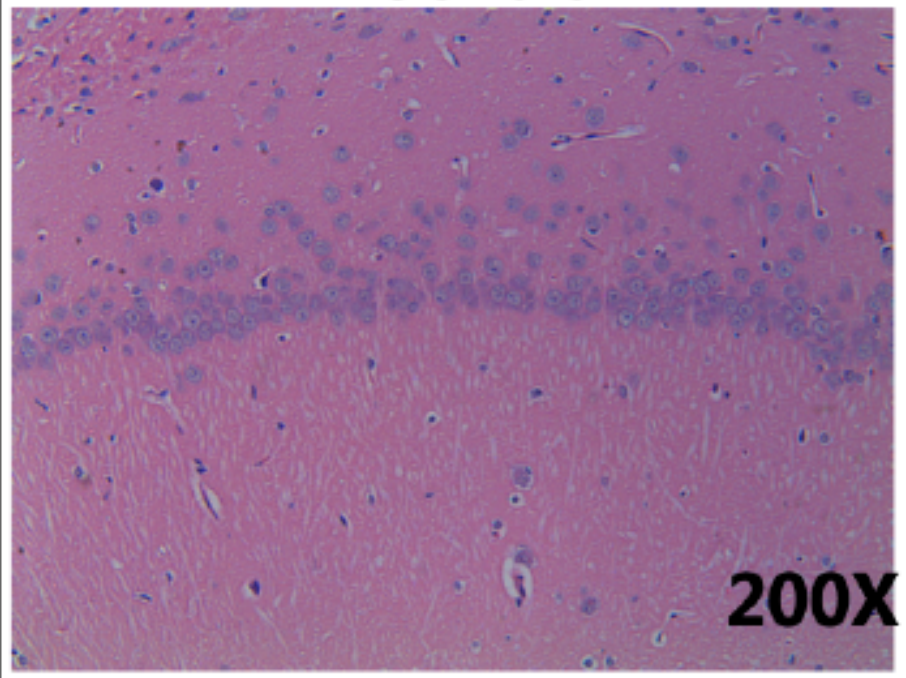

\section{Surgery}

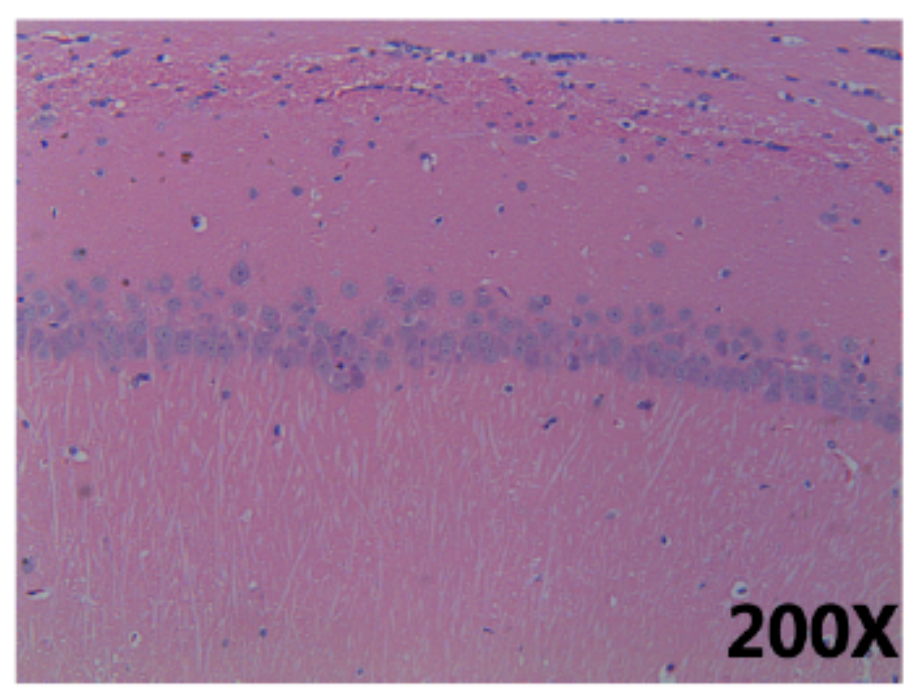

Pain

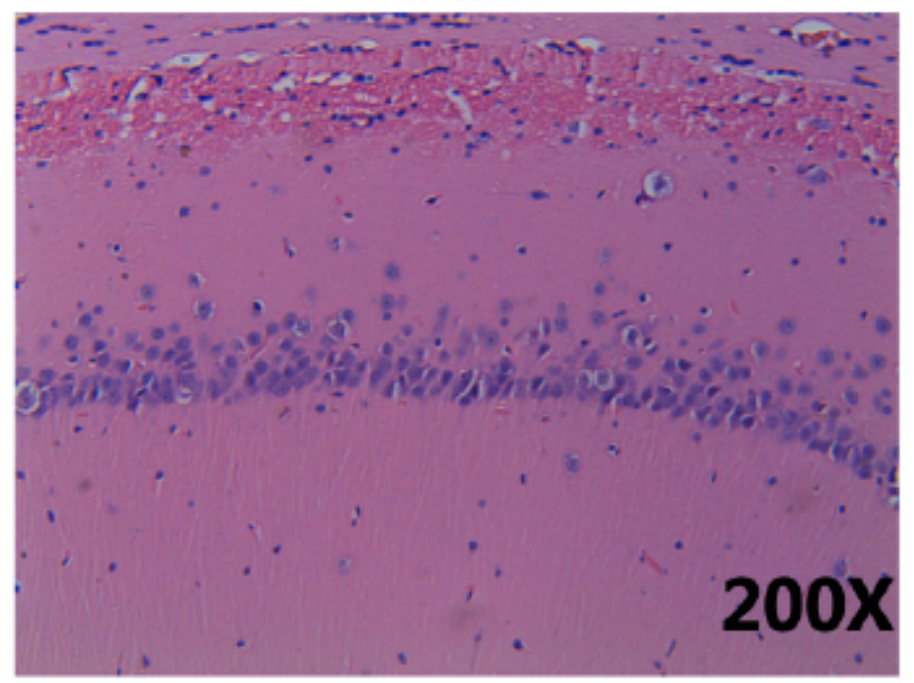

\section{Pain+Surgery}

Figure 2

Control: control group; pain: pain group; surgery: operation group; pain surgery: pain+operation group. 


\section{X}

Control

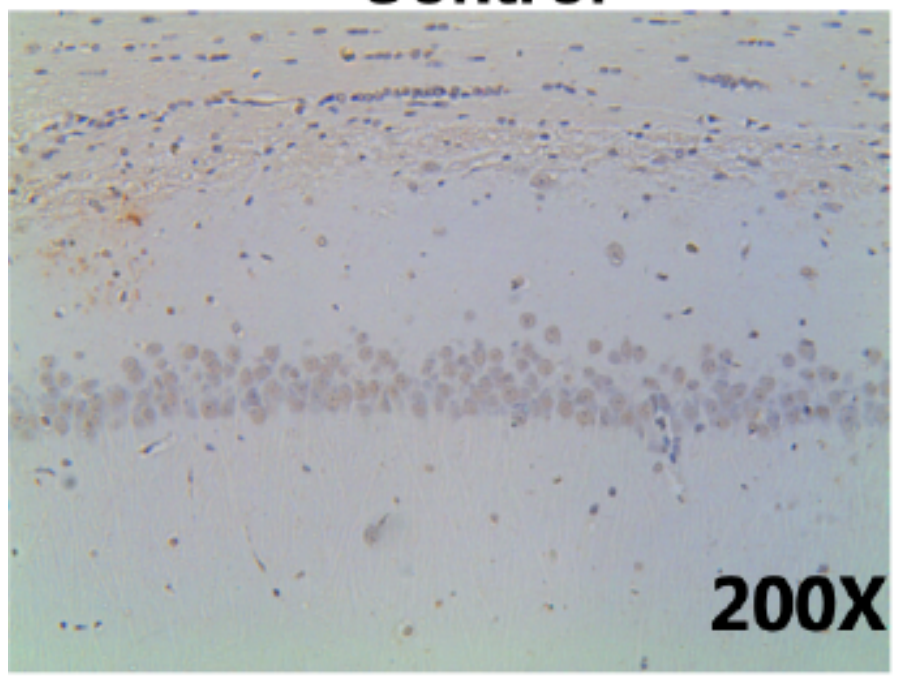

Surgery

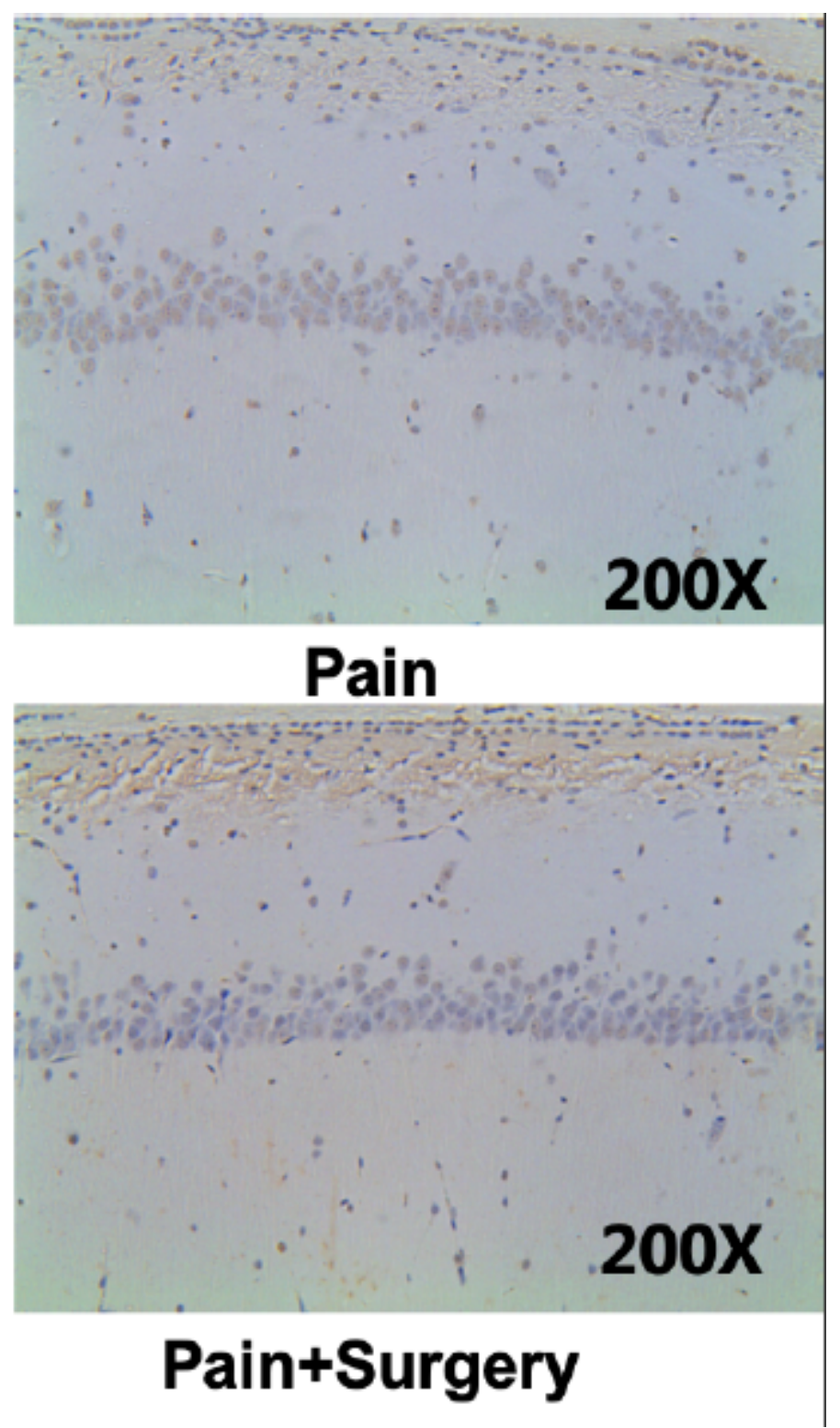

Pain+Surgery

Figure 3

Quantitative comparison of microphotographs and the apoptotic index in the hippocampus of the rats, as determined by the TUNEL method. Control: control group; pain: pain group; surgery: operation group; pain surgery: pain+operation group. 


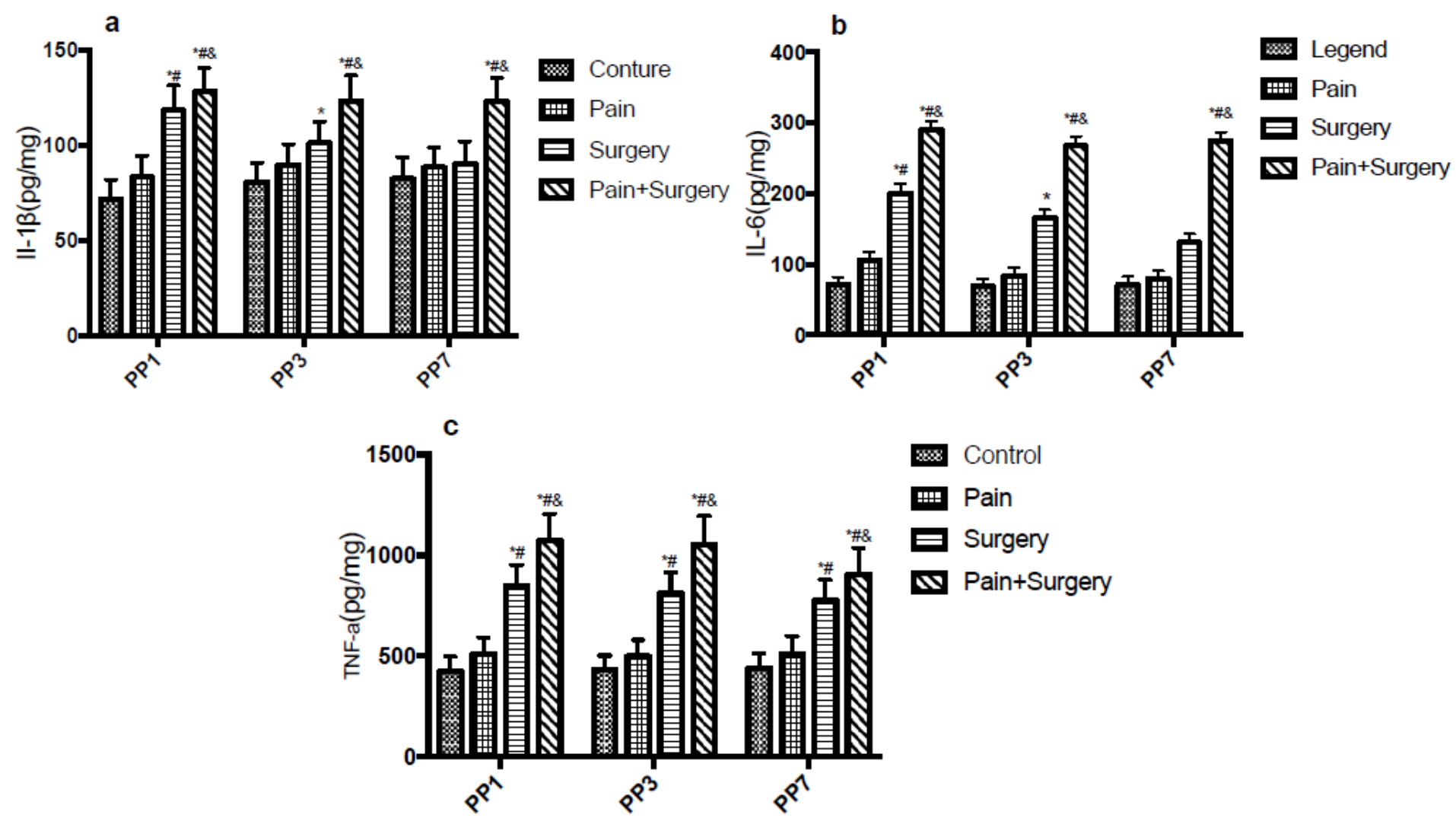

Figure 4

The expression of inflammatory cytokines after surgical trauma in the hippocampus of rats with preoperative pain. The protein contents of IL-1 $1 \beta, \mathrm{IL}-6$ and TNF- $a$ in the hippocampus of the rats were measured on the $1 \mathrm{st}$, 3rd and 7 th days after the operation. ${ }^{*} \mathrm{P}<0.05$, compared with the blank control group; \# $\mathrm{P}<0.05$ compared with the simple pain group; \& $\mathrm{P}<0.05$ compared with the simple operation group. Control: control group; pain: simple pain group; surgery: simple operation group; pain surgery: pain operation group; pp1, pp3, and pp7: 1, 3, 7 days after the operation.
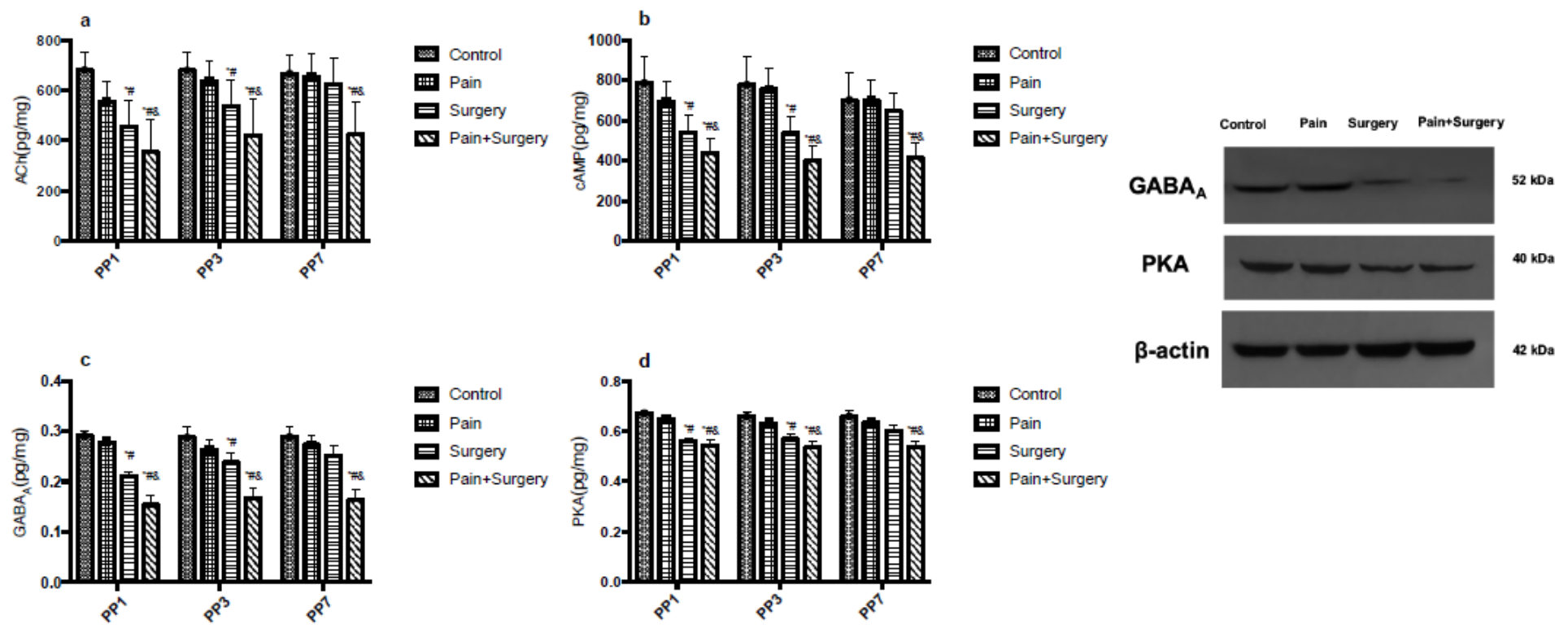


\section{Figure 5}

The expression of acetylcholine pathway factors in preoperative pain rats after surgical trauma. The protein contents of ACh, CAMP, PKA, and GABAA in the hippocampus of the rats were measured on the 1st, 3rd and 7th days after the operation. * $P<0.05$, compared with the blank control group; \# $P<0.05$, compared with the simple pain group; \& $\mathrm{P}<0.05$, compared with the simple operation group. Control: blank control group; pain: simple pain group; surgery: simple operation group; pain surgery: pain operation group; pp1, pp3, and pp7: 1, 3, and 7 days after the operation. 